\section{Energy-Based Nonlinear Control of Underactuated Euler-Lagrange Systems Subject to Impacts}

\author{
G. Hu, C. Makkar, and W. E. Dixon
}

\begin{abstract}
In this note, Lyapunov-based methods are used to design a class of energy-based nonlinear controllers to globally asymptotically stabilize/regulate an underactuated mechanical system subject to an impact collision. The impact model is considered as an elastic contact with finite stiffness. One of the difficulties in controlling impact is that the equations of motion are quite different when the system status changes from a noncontact condition to a contact condition. Another difficulty arises when an impact occurs with an underactuated system because the impact may lead to instabilities or excessive transients. An energy coupling approach is developed in this paper that is motivated by the desire to improve the transient response of the system. A Lyapunov stability analysis and numerical simulations are provided to demonstrate the stability and performance of the developed controllers.
\end{abstract}

Index Terms-Impact, Lyapunov methods, nonlinear systems.

\section{INTRODUCTION}

The control of mechanical systems subject to impact is a theoretically interesting problem with practical importance. Large stresses arise as a consequence of impact, demanding that the impact forces be properly recognized and controlled to prevent system failure. As described in [14], some useful short-duration effects such as high stresses, rapid dissipation of energy, and fast acceleration and deceleration may be achieved from low-energy sources by controlling the impact of robots operating at low force levels. Some robotic examples in which controlled contacts are required include the impact between a walking robot and the ground, the interaction of a robot manipulator with an object, multifinger grasping, and the cooperation and contact of multirobots. One of the difficulties in controlling impact is that the equations of motion are quite different when the system status changes quickly from a noncontact condition to a contact condition.

For the past decade, many researchers have addressed the modeling and control of impact [1]-[4], [8], [10]-[16]. In [2] and [11], switched controllers are given to control robotic manipulators during contact/ noncontact conditions separately. In [16], a switching control strategy is designed to guarantee the stability of the impact controller. In [12], a stable discontinuous transition controller is proposed to deal with the contact transition problem. In [10], the authors use a hybrid impedance/ time-delay controller that establishes a stable contact and achieves the desired dynamics for contact or noncontact conditions. In [13], a discontinuous Lyapunov-based control scheme is introduced to regulate the impact of a hydraulic actuator coming in contact with a nonmoving environment. In [14], a continuous proportional derivative (PD) controller is proposed to control the impact of an underactuated system where the actuators are used to stabilize the contact coordinates, and the noncontact coordinates are indirectly stabilized. In [8], static and dynamic PD controllers are proposed to address the global asymptotic

Manuscript received August 29, 2005; revised April 19, 2006 and March 15, 2007. Recommended by Associate Editor F. Bullo. This work was supported in part by the National Science Foundation under CAREER award CMS-0547448, by AFOSR under Contracts F49620-03-1-0381 and F49620-03-1-0170, and by AFRL under Contract FA4819-05-D-0011.

The authors are with the Department of Mechanical and Aerospace Engineering, University of Florida, Gainesville, FL 32611-6250 USA (e-mail: gqhu@ufl.edu; cmakkar@ufl.edu; wdixon@ufl.edu).

Color versions of one or more of the figures in this paper are available online at http://ieeexplore.iee.org.

Digital Object Identifier 10.1109/TAC.2007.904319 stabilization problem of the underactuated mechanical system subject to an impact with an elastic surface with finite stiffness, but the contacts only happen at the equilibrium points.

In this paper, we develop a new class of continuous energy-based controllers (e.g., [5] and [6]) that achieve global asymptotic stabilization/regulation of an underactuated Euler-Lagrange system subject to an elastic contact with finite stiffness. Motivation for this class of controllers is that when underactuated systems are subject to an impact, excessive transient performance may exist if natural damping is not present and the controller lacks a damping element. Hence, the developed controllers exploit the system energy to couple all the states of the system so that transients in the unactuated states will be coupled to the actuated states. This energy coupling idea is in contrast to linear controllers that do not include any state coupling. The developed stabilization result is obtained regardless of which states (i.e., actuated or unactuated) undergo an impact collision. An extension is also provided that illustrates how the actuated states can be regulated to make contact, where the unactuated states converge to the resulting closed-loop equilibrium point.

This paper is organized as follows. In Section II, the Euler-Lagrange dynamic model subject to impact is provided along with the related assumptions that are required for the control development. In Section III, the energy coupling controller is designed to globally asymptotically stabilize the generalized free motion and contact coordinates of an underactuated mechanical system subject to impact conditions, and a Lyapunov stability analysis is provided to demonstrate the stability of the developed controller. The regulation extension is provided in Section IV. In Sections V and VI, two examples and simulation results are provided to demonstrate the performance of the developed controllers. Concluding remarks are provided in the last section.

\section{DYNAMIC MODEL}

The equations of motion of an $n$-degrees-of-freedom (DOF) Euler-Lagrange system subject to an impact collision are assumed to have the following form [8], [14]:

$$
M(q) \ddot{q}+C(q, \dot{q}) \dot{q}+h(q)+R^{T} K_{c \Lambda}\left(R q-q_{c p}\right)=S u .
$$

In (1), $q(t), \dot{q}(t), \ddot{q}(t) \in \mathbb{R}^{n}$ denote the generalized position, velocity, and acceleration coordinates, $M(q) \in \mathbb{R}^{n \times n}$ denotes a positive definite inertia matrix, $C(q, \dot{q}) \dot{q} \in \mathbb{R}^{n}$ denotes the velocity-dependent force vector, $h(q) \in \mathbb{R}^{n}$ denotes a conservative force vector (e.g., spring forces, gravity), $u(t) \in \mathbb{R}^{m}$ denotes the control force/torque input, and $S \in \mathbb{R}^{n \times m}$ is a transformation matrix defined as

$$
S=\left[\begin{array}{c}
0_{(n-m) \times m} \\
I_{m}
\end{array}\right]
$$

that maps the actuator space into the generalized coordinates space, where $0_{(n-m) \times m}$ denotes an $(n-m) \times m$ matrix with all the elements equal to zero, and $I_{m} \in \mathbb{R}^{m \times m}$ is an $m \times m$ identity matrix. Also in (1), $K_{c \Lambda} \in \mathbb{R}^{r \times r}$ denotes an impact stiffness matrix defined as

$$
\begin{array}{r}
K_{c \Lambda} \triangleq \operatorname{diag}\left\{K_{c 1} \Lambda_{1}\left(q_{c 1}, q_{c p 1}\right), K_{c 2} \Lambda_{2}\left(q_{c 2}, q_{c p 2}\right), \ldots,\right. \\
\left.K_{c r} \Lambda_{r}\left(q_{c r}, q_{c p r}\right)\right\}
\end{array}
$$

where $q_{c}(t) \in \mathbb{R}^{r}$ denotes the contact coordinates that are defined as

$$
q_{c}(t) \triangleq R q(t)
$$

where $R \in \mathbb{R}^{r \times n}$ is a constant transformation matrix that maps the generalized coordinates space into the contact coordinates space and 
$q_{c p} \in \mathbb{R}^{r}$ denotes the position of the constant contact surface. In (3), the stiffness of the impact between the $i$ th contact coordinate and the corresponding contact surface is defined as $K_{c i} \in \mathbb{R}, \forall i=1, \ldots, r$, where the contact event is described by the following state-triggered discontinuous signal [8]:

$$
\Lambda_{i}\left(q_{c i}, q_{c p i}\right) \triangleq \begin{cases}0, & q_{c i}<q_{c p i} \\ 1, & q_{c i} \geq q_{c p i}\end{cases}
$$

where $q_{c i}(t)<q_{c p i}$ means the coordinate $q_{c i}(t)$ is in a noncontact condition and $q_{c i}(t) \geq q_{c p i}$ means $q_{c i}(t)$ is in contact with a surface. In the following control development, the actuated coordinates are defined by $q_{a}(t) \triangleq S^{T} q(t)$ and the corresponding unactuated states are defined as $q_{\bar{a}}(t) \in \mathbb{R}^{n-m}$.

The energy, denoted by $E(q, \dot{q})$, of the system given in (1) can be written as

$$
\begin{aligned}
E(q, \dot{q})=\frac{1}{2} \dot{q}^{T} M(q) \dot{q} & \\
+ & {\left[E_{s}(q)+\sum_{i=1}^{r} \frac{1}{2} K_{c i} \Lambda_{i}\left(q_{c i}, q_{c p i}\right)\left(q_{c i}-q_{c p i}\right)^{2}\right] }
\end{aligned}
$$

where the first term denotes the kinetic energy, $E_{s}(q) \in \mathbb{R}$ represents the potential energy of the free motion, and the second term in the brackets denotes the potential energy caused by the contact. Although (5) contains the discontinuous function $\Lambda_{i}\left(q_{c i}, q_{c p i}\right)$, the product $K_{c i} \Lambda_{i}\left(q_{c i}, q_{c p i}\right)\left(q_{c i}-q_{c p i}\right)^{2}$ is continuously differentiable. The proposed control development and stability analysis can be extended to more general contact models (for instance with damping) only if these models yield differentiable functions.

Assumption 1: The inertia matrix $M(q)$ is assumed to be symmetric and positive definite and can be upper and lower bounded by the following inequalities:

$$
a_{1}\|\xi\|^{2} \leq \xi^{T} M(q) \xi \leq a_{2}\|\xi\|^{2}, \quad \forall \xi \in \mathbb{R}^{n}
$$

where $a_{1}, a_{2} \in \mathbb{R}$ are positive constants. The following skew-symmetric relationship is also assumed to be satisfied:

$$
\xi^{T}\left(\frac{1}{2} \dot{M}(q)-C(q, \dot{q})\right) \xi=0, \quad \forall \xi \in \mathbb{R}^{n}
$$

Assumption 2: Since $h(q)$ in (1) is assumed to be a conservative force that can be derived from the potential energy, the associated power of the force is equal to the change in potential energy as

$$
\dot{E}_{s}(q)-\dot{q}^{T} h(q)=0 .
$$

Assumption 3: The system energy $E(q, \dot{q})$ is continuously differentiable, positive definite with respect to the point $\left(q^{*}, 0\right)$, where $q^{*} \in \mathbb{R}^{n}$ denotes a constant vector of equilibrium points that correspond to the minimum potential energy, and radially unbounded, and if $E(q, \dot{q}) \in$ $L_{\infty}$, then $q_{\bar{a}}(t) \in L_{\infty}$.

\section{Stabilization CONTROL DEVElOPMENT}

The stabilization problem is considered in this section, where the contact is assumed to occur at the equilibrium point (i.e., $q_{c p}=q^{*}$ ). A more general regulation problem is considered in the next section, where the generalized coordinates can be regulated to some desired setpoints if the actuation is only imposed on the generalized coordinates that make contact.

\section{A. Control Objective}

The motivation of this research is to globally asymptotically stabilize the states of an underactuated system to equilibrium points that are defined by impact and nonimpact conditions (i.e., $(q, \dot{q}) \rightarrow\left(q^{*}, 0\right)$ ). The control objective is based on the assumption that $q(t)$ and $\dot{q}(t)$ are measurable, and the states $q(t)$ and $\dot{q}(t)$ satisfy the zero-state-observability-like assumption with respect to the output $\dot{q}_{a}(t)$. The following stabilization errors, denoted by $e(t) \in \mathbb{R}^{n}, e_{c}(t) \in \mathbb{R}^{r}$, and $e_{a}(t) \in \mathbb{R}^{m}$, are introduced to quantify the control objective

$$
e=q-q^{*} \quad e_{c}=R e \quad e_{a}=S^{T} e
$$

where $e(t), e_{c}(t)$, and $e_{a}(t)$ denote the stabilization error for the generalized coordinates, contact, and actuated coordinates, respectively. Based on (9), the subsequent control development will also exploit the following zero-state-observability-like assumption.

Assumption 4: The system (1) with output $\dot{e}_{a}(t)$ is assumed to satisfy a zero-state-observability-like assumption, ${ }^{1}$ in the sense that no solution of (1) with $u(t)$ equal to a constant that is negatively proportional to $e_{a}(t)$ can stay identically in the set $\Phi=\{(q(t), \dot{q}(t)) \in$ $\left.\mathbb{R}^{n} \times \mathbb{R}^{n}:\left\|\dot{e}_{a}(t)\right\|=0\right\}$ other than the trivial solution given by $\|\dot{e}(t)\|=\|e(t)\|=0$.

Remark 1: Assumption 4 is comparable to [14, Assumptions 6 and 7], which are required in [14] to guarantee the steady-state solution is unique, $q(t)=q^{*}$ and $\|\dot{q}(t)\|=0$. Some academic mechanical examples are presented in Section V to illustrate the implications of Assumption 4.

\section{B. Open-Loop Error System}

The following expression can be obtained after taking the second time derivative of $e(t)$ and utilizing (1) and (6)

$$
\ddot{e}=M^{-1}(q)\left(S u-C(q, \dot{q}) \dot{q}-h(q)-R^{T} \bar{K}_{c \Lambda} R e\right)
$$

where

$$
\bar{K}_{c \Lambda} \triangleq \operatorname{diag}\left\{K_{c 1} \Lambda_{1}\left(e_{c 1}, 0\right), K_{c 2} \Lambda_{2}\left(e_{c 2}, 0\right), \ldots, K_{c r} \Lambda_{r}\left(e_{c r}, 0\right)\right\}
$$

After premultiplying (10) by $S^{T}$, the dynamics of the contact coordinates can be written as

$$
\ddot{e}_{a}=\frac{P(q) u+W(q, \dot{q})}{\operatorname{det}(M(q))}
$$

where the auxiliary signals $P(q) \in \mathbb{R}^{m \times m}$ and $W(q, \dot{q}) \in \mathbb{R}^{m}$ are defined as

$$
\begin{aligned}
P(q) \triangleq & S^{T} \operatorname{adj}(M(q)) S \\
W(q, \dot{q}) \triangleq & -S^{T} \operatorname{adj}(M(q)) \\
& \times\left(C(q, \dot{q}) \dot{q}+h(q)+R^{T} \bar{K}_{c \Lambda} R e\right)
\end{aligned}
$$

where $\operatorname{adj}(M)$ denotes the adjoint matrix of $M$.

Based on (5) and (9), the system energy $E(q, \dot{q})$ can be rewritten as

$$
E(q, \dot{q})=\frac{1}{2} \dot{e}^{T} M(q) \dot{e}+E_{s}(q)+\sum_{i=1}^{r} \frac{1}{2} K_{c i} \Lambda_{i}\left(e_{c i}, 0\right) e_{c i}^{2} .
$$


Taking the derivative of (15) and substituting (10) into the resulting expression yields

$$
\begin{aligned}
\dot{E}(q, \dot{q})=\dot{e}^{T}\left(\frac{1}{2} \dot{M}(q)-C(q, \dot{q})\right) \dot{e} \\
+\dot{e}^{T}(S u-h(q))+\dot{E}_{s}\left(e+q^{*}\right) .
\end{aligned}
$$

The expression in (16) can be reduced as

$$
\dot{E}(q, \dot{q})=\dot{e}^{T} S u=\dot{e}_{a}^{T} u
$$

by utilizing (7) and (8).

\section{Nonlinear Energy Coupling Controller}

Based on (12), (17), and the subsequent stability analysis, a nonlinear energy coupling controller is designed as

$$
u=[\Omega(q, \dot{q})]^{-1}\left(-K_{d} \dot{e}_{a}-K_{p} e_{a}-\frac{K_{v} W(q, \dot{q})}{\operatorname{det}(M(q))}\right)
$$

where $\Omega(q, \dot{q}) \in \mathbb{R}^{m \times m}$ is defined as

$$
\Omega(q, \dot{q}) \triangleq K_{E} E(q, \dot{q}) I_{m}+\frac{K_{v} P(q)}{\operatorname{det}(M(q))}
$$

$K_{d}, K_{p}, K_{v}$, and $K_{E} \in \mathbb{R}$ are positive constant feedback gains and $P(q)$ and $W(q, \dot{q})$ were defined in (13) and (14), respectively. Since $E(q, \dot{q})$ and $M(q)$ are assumed to be positive definite, [7, Theorem 4.2.1] can be invoked to ensure that $\Omega(q, \dot{q})$ is positive definite; hence, $\Omega(q, \dot{q})$ is invertible. After substituting (18) into (12), the closed-loop error system for $\ddot{e}_{a}(t)$ can be obtained as

$$
\begin{aligned}
\ddot{e}_{a}= & \frac{1}{\operatorname{det}(M(q))} P(q)[\Omega(q, \dot{q})]^{-1} \\
& \times\left(-K_{d} \dot{e}_{a}-K_{p} e_{a}-\frac{K_{v} W(q, \dot{q})}{\operatorname{det}(M(q))}\right)+\frac{W(q, \dot{q})}{\operatorname{det}(M(q))} .
\end{aligned}
$$

After substituting (18) into (17), the derivative for the system energy can be obtained as

$$
\dot{E}(q, \dot{q})=\dot{e}_{a}^{T}[\Omega(q, \dot{q})]^{-1}\left(-K_{d} \dot{e}_{a}-K_{p} e_{a}-\frac{K_{v} W(q, \dot{q})}{\operatorname{det}(M(q))}\right) .
$$

Theorem 1: The equilibrium points of the system in (1) with the controller defined in (18) are globally asymptotically stable in the sense that

$$
q(t) \rightarrow q^{*} \quad \text { and } \quad \dot{q}(t) \rightarrow 0 \quad \text { as } \quad t \rightarrow \infty
$$

Proof: Let $V_{1}(e, \dot{e}) \in \mathbb{R}$ denote the following continuously differentiable, positive definite, radially unbounded function (i.e., a Lyapunov function candidate)

$$
V_{1}(e, \dot{e})=\frac{1}{2} K_{E} E^{2}(q, \dot{q})+\frac{1}{2} K_{p} e_{a}^{T} e_{a}+\frac{1}{2} K_{v} \dot{e}_{a}^{T} \dot{e}_{a} .
$$

Based on the closed-loop error systems in (20) and (21), the time derivative of (23) can be expressed as

$$
\begin{aligned}
\dot{V}_{1}(e, \dot{e})= & K_{E} E(q, \dot{q}) \dot{e}_{a}^{T}[\Omega(q, \dot{q})]^{-1} \\
& \times\left(-K_{d} \dot{e}_{a}-K_{p} e_{a}-\frac{K_{v} W(q, \dot{q})}{\operatorname{det}(M(q))}\right) \\
& +K_{p} \dot{e}_{a}^{T} e_{a}+K_{v} \dot{e}_{a}^{T} \frac{W(q, \dot{q})}{\operatorname{det}(M(q))} \\
& -\frac{K_{v} \dot{e}_{a}^{T}}{\operatorname{det}(M(q))} P(q)[\Omega(q, \dot{q})]^{-1} \\
& \times\left(K_{d} \dot{e}_{a}+K_{p} e_{a}+\frac{K_{v} W(q, \dot{q})}{\operatorname{det}(M(q))}\right) .
\end{aligned}
$$

The expression in (24) can be rewritten as

$$
\begin{aligned}
\dot{V}_{1}(e, \dot{e})= & \dot{e}_{a}^{T}\left(K_{E} E(q, \dot{q}) I_{m}+K_{v} \frac{P(q)}{\operatorname{det}(M(q))}\right) \\
& \times[\Omega(q, \dot{q})]^{-1}\left(-K_{d} \dot{e}_{a}-K_{p} e_{a}-\frac{K_{v} W(q, \dot{q})}{\operatorname{det}(M(q))}\right) \\
& +K_{p} \dot{e}_{a}^{T} e_{a}+K_{v} \dot{e}_{a}^{T} \frac{W(q, \dot{q})}{\operatorname{det}(M(q))} .
\end{aligned}
$$

The expression in (25) can be simplified as

$$
\dot{V}_{1}(e, \dot{e})=-K_{d} \dot{e}_{a}^{T} \dot{e}_{a} \leq 0
$$

where (19) was utilized (i.e., $\dot{V}_{1}(e, \dot{e})$ is negative semidefinite). From (23) and (26), the origin of the closed-loop system is stable in the sense of Lyapunov and $V_{1}(e, \dot{e}) \in \mathcal{L}_{\infty}$; hence, $E(q, \dot{q}), e_{a}(t)$, and $\dot{e}_{a}(t) \in$ $\mathcal{L}_{\infty}$. Since $E(q, \dot{q}) \in \mathcal{L}_{\infty},(15)$ can be used to prove that $\dot{e}(t) \in \mathcal{L}_{\infty}$ and Assumption 3 can be used to conclude that $q_{\bar{a}}(t) \in \mathcal{L}_{\infty}$; hence, $e(t) \in \mathcal{L}_{\infty}$. Since $e(t), \dot{e}(t) \in \mathcal{L}_{\infty},(9)$ can be used to prove that $q(t), \dot{q}(t) \in \mathcal{L}_{\infty}$. The definitions in (13), (14), and (19) can now be used to prove that $P(q), W(q, \dot{q})$, and $\Omega(q, \dot{q}) \in \mathcal{L}_{\infty}$. The proceeding arguments can be used along with (18) to prove that $u(t) \in \mathcal{L}_{\infty}$.

Based on the fact that all of the closed-loop signals remain bounded, LaSalle's invariance theorem can now be utilized to prove Theorem 1. To this end, let $\bar{\Phi}$ denote the following set:

$$
\bar{\Phi}=\left\{(e, \dot{e}) \in \mathbb{R}^{n} \times \mathbb{R}^{n}: \dot{V}_{1}(e, \dot{e})=0\right\} .
$$

In the set $\bar{\Phi}$, it is clear from (26) that

$$
\dot{e}_{a}(t)=0 \quad \ddot{e}_{a}(t)=0
$$

and hence, from (21), we can conclude that

$$
\dot{E}(q, \dot{q})=0 .
$$

The expressions in (21), (23), and (27)-(29) can be used to prove that $e_{a}(t), E(q, \dot{q})$, and $V_{1}(e, \dot{e})$ are constant. To prove that $u(t)$ is constant in $\bar{\Phi}$, we rewrite (12) as

$$
\frac{1}{\operatorname{det}(M(q))} W(q, \dot{q})=\ddot{e}_{a}-\frac{1}{\operatorname{det}(M(q))} P(q) u
$$

and substitute (30) into (18) to obtain the following expression:

$$
\begin{aligned}
u=[\Omega(q, \dot{q})]^{-1}\left(-K_{d} \dot{e}_{a}-\right. & K_{p} e_{a}-K_{v} \\
& \left.\times\left(\ddot{e}_{a}-\frac{1}{\operatorname{det}(M(q))} P(q) u\right)\right) .
\end{aligned}
$$

After multiplying $\Omega(q, \dot{q})$ on both sides of (31), the following simplified relationship can be developed:

$$
u=\frac{1}{K_{E} E(q, \dot{q})}\left(-K_{d} \dot{e}_{a}-K_{p} e_{a}-K_{v} \ddot{e}_{a}\right)
$$

Since $e_{a}(t)$ and $E(q, \dot{q})$ have been proven to be constant in $\bar{\Phi},(32)$ can be used to conclude that $u(t)$ is equal to the following constant:

$$
u=-\frac{1}{K_{E} E(q, \dot{q})} K_{p} e_{a}
$$

Since $\dot{e}_{a}(t)$ is equal to zero, $u(t)$ and $E(q, \dot{q})$ are constant and satisfy (33), and the zero-state-observability-like assumption (i.e., Assumption 4) can be used to conclude the result in (22).

Remark 2: One advantage with the controller in (18) proposed in this paper is that the stability conditions in (26) do not depend on the contact stiffness, contrary to some conditions that may be found in the literature. However, the resulting controller may yield high gains because of the inclusion of the contact stiffness in the control input. 
Remark 3: In Section V, two examples are provided to illustrate the physical implications of the zero-state-observability-like assumption.

\section{REGULATION EXTENSION}

In the previous section, a nonlinear controller was designed to stabilize the generalized coordinates to the equilibrium points in both contact and free motion conditions. In this section, the control objective is extended from a stabilization problem to a regulation problem when the actuation is only imposed on the contact coordinates (i.e., $R^{T}=S$ ). The actuated coordinates can be asymptotically regulated to some constant desired position where contact occurs. To satisfy this objective, the regulation problem is transformed into a stabilization problem for a new virtual system that has dynamics similar to the original system. The design method in Section III is then used to control the transformed system.

For the control objective in this section, let $q_{d} \in \mathbb{R}^{n}$ denote the known constant desired setpoints, where $q_{a d} \in \mathbb{R}^{m}$ denotes a subset of $q_{d}$ corresponding to the desired positions of the actuated coordinates $q_{a}(t)$. The dynamics given in (1) can be expressed in terms of the desired setpoints as

$$
M\left(q_{d}\right) \ddot{q}_{d}+C\left(q_{d}, \dot{q}_{d}\right) \dot{q}_{d}+h\left(q_{d}\right)+R^{T} \tilde{K}_{c \Lambda}\left(R q_{d}-q_{c p}\right)=S u_{d}
$$

where $\tilde{K}_{c \Lambda}=\operatorname{diag}\left\{K_{c 1} \Lambda_{1}\left(q_{c 1 d}, q_{c p 1}\right), K_{c 2} \Lambda_{2}\left(q_{c 2 d}, q_{c p 2}\right), \ldots\right.$, $\left.K_{c r} \Lambda_{r}\left(q_{c r d}, q_{c p r}\right)\right\}, u_{d} \in \mathbb{R}^{m}$ denotes a known residual force, and $q_{\text {cid }}$ denotes the constant desired contact coordinates $\forall i=1,2, \ldots, r$. Since $q_{d}$ is a constant and $R q_{d}$ is assumed to equal $q_{c p}$ for the regulation problem, (34) can be simplified as

$$
h\left(q_{d}\right)=S u_{d} .
$$

Based on (1) and (35), the following open-loop error system can be obtained:

$$
M(q) \ddot{q}+C(q, \dot{q}) \dot{q}+h(q)-h\left(q_{d}\right)+R^{T} K_{c \Lambda}\left(R q-q_{c p}\right)=S\left(u-u_{d}\right) .
$$

The energy of the system, denoted by $E(q, \dot{q})$, given in (36) can be written as

$$
\begin{aligned}
E(q, \dot{q})= & \frac{1}{2} \dot{q}^{T} M(q) \dot{q} \\
& +\left[E_{s v}(q)+\sum_{i=1}^{r} \frac{1}{2} K_{c i} \Lambda_{i}\left(q_{c i}, q_{c p i}\right)\left(q_{c i}-q_{c p i}\right)^{2}\right]
\end{aligned}
$$

where $E_{s v}(q)$ denotes the known potential energy with respect to the conservative forces $h(q)$ and $h\left(q_{d}\right)$. Based on (36), the time derivative of (37) can be obtained as

$$
\dot{E}(q, \dot{q})=\dot{e}^{T} S\left(u-u_{d}\right)=\dot{e}_{a}^{T}\left(u-u_{d}\right)
$$

where the regulation error $e(t) \in \mathbb{R}^{n}$ and the regulation error for the actuated coordinates $e_{a}(t) \in \mathbb{R}^{m}$ are defined as

$$
e=q-q_{d} \quad e_{a}=S^{T} e=q_{a}-q_{a d} .
$$

Based on the subsequent development, the force control input $u(t)$ is designed as follows for the regulation problem:

$$
u=[\Omega(q, \dot{q})]^{-1}\left(-K_{d} \dot{e}_{a}-K_{p} e_{a}-\frac{K_{v} W(q, \dot{q})}{\operatorname{det}(M(q))}\right)+u_{d}
$$

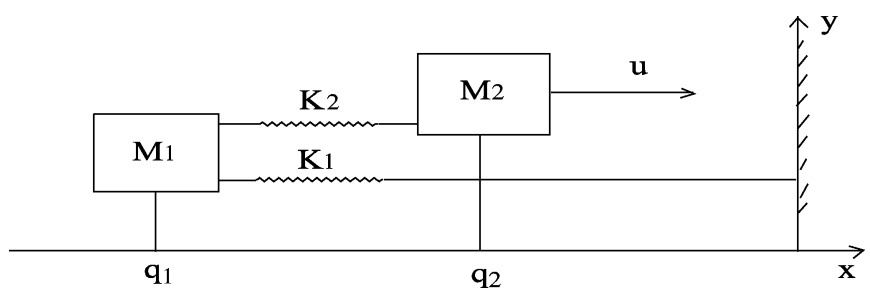

Fig. 1. The mass spring system represents an academic example of a general underactuated Euler-Lagrange system with contact and noncontact states.

where $P(q)$ and $\Omega(q, \dot{q})$ are defined in (13) and (19), respectively, and $W(q, \dot{q})$ is now defined as

$$
\begin{aligned}
W(q, \dot{q})=-S^{T} \operatorname{adj}(M(q)) & (C(q, \dot{q}) \dot{q}+h(q) \\
& \left.-h\left(q_{d}\right)+R^{T} K_{c \Lambda}\left(R q-q_{c}^{*}\right)\right) .
\end{aligned}
$$

Theorem 2: Under Assumptions 1-4, the controller in (39) yields globally asymptotic regulation in the sense that

$$
q(t) \rightarrow q_{d} \quad \text { and } \quad \dot{q}(t) \rightarrow 0 \quad \text { as } \quad t \rightarrow \infty .
$$

Proof: See the proof for Theorem 1.

\section{EXAMPLES}

In this section, two examples are given to show the performance of the proposed control design method. These examples also illustrate the physical implications of the zero-state-observability-like assumption.

\section{A. Example 1}

An example of the class of systems considered in this paper can be described by the mass spring system introduced in [8] that is depicted in Fig. 1. As the figure shows, the system consists of two masses $M_{1}$ and $M_{2}$ that are coupled to each other and to a fixed surface through springs. The objective for this academic example is to design an input force $u(t)$ so that $M_{2}$ is regulated to be in contact with the surface of a fixed object while $M_{1}$ is regulated to a stable noncontact equilibrium point.

The equations of motion of the system are given by the following differential equations:

$$
\begin{array}{r}
M_{1} \ddot{q}_{1}+\left(K_{1}+K_{2}\right)\left(q_{1}-q_{1}^{*}\right)-K_{2}\left(q_{2}-q_{2}^{*}\right)=0 \\
M_{2} \ddot{q}_{2}-K_{2}\left(q_{1}-q_{1}^{*}\right)+\left(K_{2}+K_{c 1} \Lambda_{1}\left(q_{2}, q_{2}^{*}\right)\right)\left(q_{2}-q_{2}^{*}\right)=u
\end{array}
$$

where $q_{1}(t), q_{2}(t)$ denote the positions of $M_{1}$ and $M_{2}$, respectively, and $q_{1}^{*}, q_{2}^{*}$ denote the equilibrium points of $M_{1}$ and $M_{2}$, respectively. After utilizing (9), the dynamics in (42) can be expressed as

$$
\begin{aligned}
M_{1} \ddot{e}_{1}+\left(K_{1}+K_{2}\right) e_{1}-K_{2} e_{2} & =0 \\
M_{2} \ddot{e}_{2}-K_{2} e_{1}+K_{2} e_{2}+K_{c 1} \Lambda_{1}\left(e_{2}, 0\right) e_{2} & =u .
\end{aligned}
$$

Based on (43), it is straightforward to verify that Assumption 1 is satisfied.

The total energy of the system is given by

$$
\begin{aligned}
E=\frac{1}{2} K_{1} e_{1}^{2}+\frac{1}{2} K_{2}\left(e_{1}-e_{2}\right)^{2}+ & \frac{1}{2} K_{c 1} \Lambda_{1}\left(e_{2}, 0\right) e_{2}^{2} \\
& +\frac{1}{2} M_{1} \dot{e}_{1}^{2}+\frac{1}{2} M_{2} \dot{e}_{2}^{2}
\end{aligned}
$$

where the first two terms in (44) denote the potential energy of the free motion system, the third term denotes the potential energy generated by contact, and the last two terms represent the kinetic energy. Based on 


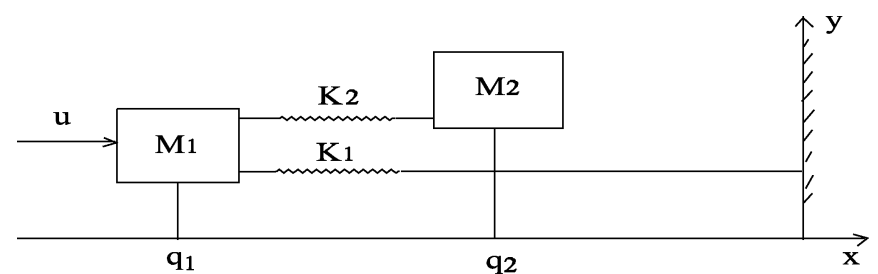

Fig. 2. The mass spring system represents an academic example of a general underactuated Euler-Lagrange system where contact coordinates are different to actuated coordinates.

(42), the matrix $S$ and the definitions in (13) and (14) can be expressed as

$$
\begin{aligned}
S & =\left[\begin{array}{ll}
0 & 1
\end{array}\right]^{T} \\
P & =M_{1} \\
W & =M_{1}\left(K_{2} e_{1}-K_{2} e_{2}-K_{c 1} \Lambda_{1}\left(e_{2}, 0\right) e_{2}\right) .
\end{aligned}
$$

Based on (42) and (45)-(47), the energy-based controller given in (18) can be expressed as

$$
\begin{aligned}
u=\frac{M_{2}}{K_{E} E M_{2}+} & K_{v} \\
& \left(-K_{d} \dot{e}_{2}-K_{p} e_{2}\right. \\
& \left.-\frac{K_{v}}{M_{2}}\left(K_{2}\left(e_{1}-e_{2}\right)-K_{c 1} \Lambda_{1}\left(e_{2}, 0\right) e_{2}\right)\right) .
\end{aligned}
$$

The conservative spring force for the example mass spring problem is given by

$$
h(q)=\left[\begin{array}{c}
\left(K_{1}+K_{2}\right)\left(q_{1}-q_{1}^{*}\right)-K_{2}\left(q_{2}-q_{2}^{*}\right) \\
-K_{2}\left(q_{1}-q_{1}^{*}\right)+K_{2}\left(q_{2}-q_{2}^{*}\right)
\end{array}\right]
$$

and the potential energy of the system can also be written as

$$
E_{s}=\frac{1}{2} K_{1}\left(q_{1}-q_{1}^{*}\right)^{2}+\frac{1}{2} K_{2}\left(q_{1}-q_{2}-q_{1}^{*}+q_{2}^{*}\right)^{2}
$$

The differential expression in (8) of Assumption 2 is satisfied for (49) and (50). Based on (44), it is clear that Assumption 3 is also satisfied.

To verify Assumption 4, we consider the system in (43). If $\dot{e}_{2}(t)$ is equal to zero, then $e_{2}(t)$ is constant. Hence, the forces that act on $M_{1}$ are balanced. If $e_{1}(t)$ changes, then the force on the spring $K_{2}$ changes, which causes $M_{2}$ to move. If $M_{2}$ moves, then $e_{2}(t)$ and $u(t)$ will not remain constant, which is a contradiction (hence, $e_{1}(t)$ is also constant). Based on the form of (33) and (48), the control input can be expressed as $u(t)=-c_{1} e_{2}(t)$, where $c_{1}$ is a positive constant. Since $e_{1}(t)$ and $e_{2}(t)$ are constant, (43) can be expressed as

$$
\begin{array}{r}
K_{1} e_{1}+K_{2}\left(e_{1}-e_{2}\right)=0 \\
-c_{1} e_{2}-K_{c 1} \Lambda_{1}\left(e_{2}, 0\right) e_{2}+K_{2}\left(e_{1}-e_{2}\right)=0 .
\end{array}
$$

The expressions in (51) and (52) are linearly independent; therefore, $e_{1}(t)=e_{2}(t)=0$ [i.e., the zero-state-observability-like assumption (Assumption 4) is satisfied].

\section{B. Example 2}

In the previous example, the actuation is applied to the contact coordinates. In this example, the contact coordinates are not the same as actuated coordinates. Specifically, as indicated in Fig. 2, the actuation is applied to $M_{1}$, whereas the contact occurs between $M_{2}$ and the surface.
The equations of motion of the system in Fig. 2 are

$$
\begin{array}{r}
M_{1} \ddot{q}_{1}+\left(K_{1}+K_{2}\right)\left(q_{1}-q_{1}^{*}\right)-K_{2}\left(q_{2}-q_{2}^{*}\right)=u \\
M_{2} \ddot{q}_{2}-K_{2}\left(q_{1}-q_{1}^{*}\right)+\left(K_{2}+K_{c 1} \Lambda_{1}\left(q_{2}, q_{2}^{*}\right)\right)\left(q_{2}-q_{2}^{*}\right)=0 .
\end{array}
$$

After utilizing (9), the dynamics in (53) can be expressed as

$$
\begin{aligned}
M_{1} \ddot{e}_{1}+\left(K_{1}+K_{2}\right) e_{1}-K_{2} e_{2} & =u \\
M_{2} \ddot{e}_{2}-K_{2} e_{1}+K_{2} e_{2}+K_{c 1} \Lambda_{1}\left(e_{2}, 0\right) e_{2} & =0 .
\end{aligned}
$$

Based on (54), the matrix $S$ and the definitions in (13) and (14) can be expressed as

$$
\begin{aligned}
S & =\left[\begin{array}{ll}
1 & 0
\end{array}\right]^{T} \quad P=M_{2} \\
W & =-M_{2}\left(\left(K_{1}+K_{2}\right) e_{1}-K_{2} e_{2}\right) .
\end{aligned}
$$

Based on (54)-(56), the energy-based controller given in (18) can be expressed as

$$
\begin{aligned}
u=\frac{M_{1}}{K_{E} E M_{1}+K_{v}}\left(-K_{d} \dot{e}_{1}-K_{p} e_{1}\right. & \\
& \left.-\frac{K_{v}}{M_{1}}\left(K_{2} e_{2}-\left(K_{1}+K_{2}\right) e_{1}\right)\right) .
\end{aligned}
$$

Since the models depicted in Figs. 1 and 2 have the same structure, Assumptions 1-3 can be satisfied as stated in the previous example. Likewise, using the same arguments as in the previous example, the zero-state-observability assumption can be verified.

\section{NUMERICAL SimULATION}

To illustrate the performance of the energy-based controller in (18), numerical simulations were performed for the example system depicted in Fig. 1. The performance of the proposed energy coupling controller is compared with the following PD controller:

$$
u=-\left(K_{d} \dot{e}_{2}+K_{p} e_{2}\right) .
$$

For the simulation, the physical parameters of the mass spring system were selected as

$$
\begin{aligned}
M_{1} & =1[\mathrm{~kg}], \quad M_{2}=1[\mathrm{~kg}] \\
K_{1} & =10^{3}[\mathrm{~N} / \mathrm{m}], \quad K_{2}=5 \times 10^{3}[\mathrm{~N} / \mathrm{m}] \\
K_{c 1} & =10^{5}[\mathrm{~N} / \mathrm{m}] .
\end{aligned}
$$

The equilibrium positions of $M_{1}$ and $M_{2}$ were set to the following values:

$$
\left[\begin{array}{ll}
q_{1}^{*} & q_{2}^{*}
\end{array}\right]^{T}=\left[\begin{array}{ll}
-0.5 & 0
\end{array}\right]^{T}[m]
$$

and the initial conditions for $q_{1}(t)$ and $q_{2}(t)$ were selected as

$$
\left[q_{1}(0) \quad q_{2}(0)\right]^{T}=\left[\begin{array}{ll}
-1.0 & -0.5
\end{array}\right]^{T}[m] .
$$

For the continuous energy-based controller proposed in (18) and the PD controller in (58), the resulting control gains from each controller are given in Table I. The resulting positions of $M_{1}$ and $M_{2}$ and the input force are shown in Fig. 3 for the PD control law and Fig. 4 for the energy coupling control law. The performance of the proposed controller in (18) and the PD controller in (58) is quantified in Table II in terms of the settling time $T_{s}$ (determined as the time required to reach the equilibrium point where $\left.\left|e_{i}(t)\right|<0.01, i=1,2\right)$ and the integral of 
TABLE I

CONTROL GAINS FOR THE CONTINUOUS ENERGY-BASEd CONTROLLER AND A PD CONTROLLER

\begin{tabular}{|l|l|l|l|l|}
\hline Control gains & $K_{p}$ & $K_{d}$ & $K_{E}$ & $K_{v}$ \\
\hline Controller in (58) & 100 & 30 & NA & NA \\
\hline Controller in (18) & 100 & 300 & 0.03 & 1.6 \\
\hline
\end{tabular}
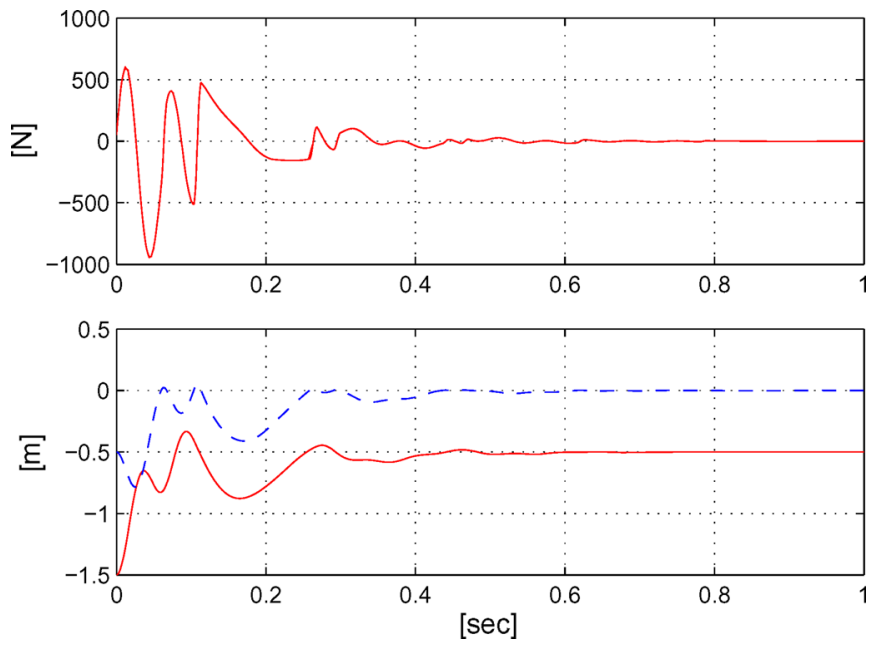

Fig. 3. Results for the PD controller where contact is shown. The top figure depicts the control input where the steady-state control force is zero for the stabilization problem. In the bottom figure, the solid curve is the position of $M_{1}$ and the dashed curve is the position of $M_{2}$.
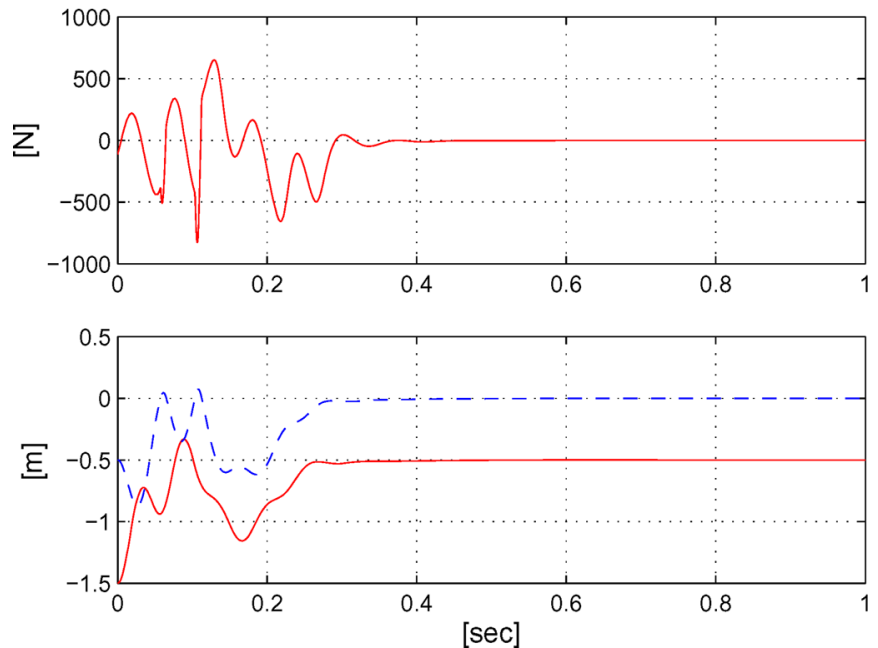

Fig. 4. Results for the energy-based coupling controller where contact is shown. The top figure depicts the control input where the steady-state control force is zero for the stabilization problem. In the bottom figure, the solid curve is the position of $M_{1}$ and the dashed curve is the position of $M_{2}$.

the error squared. The respective control input for each controller is provided in Table III in terms of the maximum force and the integral of the force squared.

Based on the results given in Tables II and III and illustrated in Figs. 3 and 4 , it is clear that the energy coupling control laws exhibit improved transient performance (while requiring less control effort) when compared to the PD controller in (58). The improved performance supports the hypothesis that improved response will be obtained by incorporating the system energy in the control development. By including the system energy in the control design, the states become coupled,
TABLE II

Performance Comparison For the Continuous Energy-BASED CONTROLLER AND A PD CONTROLLER

\begin{tabular}{|l|l|l|l|}
\hline Performance & $T_{s}[\mathrm{sec}]$ & $\int_{0}^{1} e_{1}^{2}(\sigma) d \sigma$ & $\int_{0}^{1} e_{2}^{2}(\sigma) d \sigma$ \\
\hline Controller in (58) & 0.58 & 0.0274 & 0.0325 \\
\hline Controller in (18) & 0.28 & 0.0429 & 0.0554 \\
\hline
\end{tabular}

TABLE III

CONTROL EFForT COMPARISON FOR THE CONTINUOUS ENERGY-BASED CONTROLLER AND A PD CONTROLLER

\begin{tabular}{|l|l|l|}
\hline Control effort & $\max |u|[\mathrm{N}]$ & $\int_{0}^{1} u^{2}(\sigma) d \sigma$ \\
\hline Controller in (58) & 900 & $0.33 \times 10^{5}$ \\
\hline Controller in (18) & 800 & $0.31 \times 10^{5}$ \\
\hline
\end{tabular}

whereas the PD controller only uses the position and velocity information of the contact states.

\section{CONCLUSION}

A continuous energy-based control method is proposed to stabilize/ regulate the states of a general class of underactuated Euler-Lagrange system in free motion and in contact. The efforts in this paper are inspired by the heuristic idea that improved transient response will result from using the system energy to couple the states in the controller. The class of controllers is developed through a Lyapunov-based stability analysis. Two academic examples are provided to illustrate the theoretical development, and numerical simulations are provided to demonstrate the stability and performance of the developed controllers. The simulation results support the hypothesis that the energy-based controllers result in faster transient response than a PD controller. In future efforts, we hope to incorporate some transient performance index in the control design and stability analysis to develop analytical support of the idea that the developed class of energy-based controllers leads to improved transient response over other classes of controllers that do not couple the underactuated states (i.e., a PD controller). Furthermore, future efforts will focus on an adaptive and output feedback extensions of the developed controllers to reduce the required amount of state and model knowledge.

\section{REFERENCES}

[1] B. Brogliato, S. I. Niculescu, and P. Orhant, "On the control of finite-dimensional mechanical systems with unilateral constraints," IEEE Trans. Autom. Control, vol. 42, no. 2, pp. 200-215, 1997.

[2] B. Brogliato and P. Orhant, "Contact stability analysis of a one degree-of-freedom robot," Dynamics Contr., vol. 8, pp. 37-53, 1998.

[3] B. Brogliato and A. Zavala Rio, "On the control of complementaryslackness juggling mechanical systems," IEEE Trans. Autom. Control, vol. 45, no. 2, pp. 235-246, 2000.

[4] D. Chiu and S. Lee, "Robust jump impact controller for manipulators," in Proc. 1995 IEEE/RSJ Int. Conf. Intell. Robots Syst., Pittsburgh, PA, 1995, vol. 1, pp. 299-304.

[5] I. Fantoni, R. Lozano, and M. W. Spong, "Energy based control of the pendubot," IEEE Trans. Autom. Control, vol. 45, no. 4, pp. 725-729, 2000.

[6] Y. Fang, W. E. Dixon, D. M. Dawson, and E. Zergeroglu, "Nonlinear coupling control laws for a 3-DOF overhead crane system," IEEE/ASME Trans. Mechatronics, vol. 8, no. 3, pp. 418-423, 2003.

[7] G. H. Golub and C. F. Van Loan, Matrix Computations 3rd Edition. Baltimore, MD: Johns Hopkins Univ. Press, 1996.

[8] M. Indri and A. Tornambe, "Control of under-actuated mechanical systems subject to smooth impacts," in Proc. IEEE Conf. Decision Contr., Atlantis, Paradise Island, Bahamas, 2004, pp. 1228-1233.

[9] H. K. Khalil, Nonlinear Systems, 3rd ed. Englewood Cliffs, NJ: Prentice-Hall, 2002. 
[10] E. Lee et al., "Bang-bang impact control using hybrid impedance timedelay control," IEEE/ASME Trans. Mechatronics, vol. 8, no. 2, pp. 272-277, 2003.

[11] J. K. Mills and D. M. Lokhorst, "Stability and control of robotic manipulators during contact/noncontact task transition," IEEE Trans. Robot. Automat., vol. 9, no. 3, pp. 335-345, 1993.

[12] P. R. Pagilla and B. Yu, "A stable transition controller for constrained robots," IEEE/ASME Trans. Mechatronics, vol. 6, no. 1, pp. 65-74, 2001.

[13] P. Sekhavat, Q. Wu, and N. Sepehri, "Impact control in hydraulic actuators with friction: Theory and experiments," in Proc. Amer. Contr. Conf., Boston, MA, 2004, pp. 4432-4437.

[14] A. Tornambe, "Modeling and control of impact in mechanical systems: Theory and experimental results," IEEE Trans. Autom. Control, vol. 44, no. 2, pp. 294-309, 1999.

[15] R. Volpe and P. Khosla, "A theoretical and experimental investigation of impact control for manipulators," Int. J. Robot. Res., vol. 12, no. 4, pp. 352-365, 1993.

[16] Y. Wu, T. J. Tarn, N. Xi, and A. Isidori, "On robust impact control via positive acceleration feedback for robot manipulators," in Proc. IEEE Int. Conf. Robot. Automat., Albuquerque, NM, 1996, pp. 1891-1896.

\section{Repetitive Control of Positive Real Systems via Delayed Feedback Is Lyapunov Asymptotically Stable}

\author{
Pasquale Lucibello
}

\begin{abstract}
In this paper, we are concerned with the analysis of linear infinite-dimensional control systems that should be able to compensate and/or track signals that are periodic. Adopting the name given in the seminal paper by Hara et al., we call them repetitive controllers. We analyze the asymptotic stability in the Lyapunov sense of finite-dimensional positive real plants coupled with pure delays. For this class of systems, we initially prove convergence in the weak topology to later deduce convergence in the strong one.
\end{abstract}

Index Terms-Control systems, delay systems, linear systems, Lyapunov methods.

\section{INTRODUCTION}

In this paper, we deal with the problem of designing a controller for a finite-dimensional system that has to track signals and reject disturbances that are periodic. For example, such a kind of problem is faced in the case of mechanical systems, such as industrial robots and numerical machines, that have to execute repetitive commands or when disturbances depending on the frequency of the power supply have to be rejected [1]; in the design of high-precision tracking control systems for digital video disk players [2]; in the precise speed control for ultrasonic motors [3]; and when dealing with high-accuracy magnet power supply for proton synchrotron [4]. This type of control is usually referred to as repetitive control, a name first adopted by Hara et al. [1], who have studied a controller incorporating a pure delay.

In [5], an adaptive control scheme has been proposed that incorporates a generator of the control signal of the integral type. This scheme suffers the shortcoming that a state trajectory to be tracked must be known, and not just the output trajectory.

The focus of this paper is on a very peculiar aspect: the asymptotic stability in the Lyapunov sense of the controller proposed in [1] when

Manuscript received September 20, 2004; revised October 17, 2005, July 13, 2006, and June 12, 2007. Recommended by Associate Editor E. Jonckheere.

The author is with Società Gestioni Impianti Nucleari, 00184 Roma, Italy (e-mail: lucibello@sogin.it).

Digital Object Identifier 10.1109/TAC.2007.904320 coupled with a positive real system (see, e.g., [6] for an introduction to this class of systems and [7] for an explanation of the class of control systems that can be transformed in a real positive one). The reason for such a narrow focus is due to the fact that this coupled system belongs to the class of neutral differential-difference equations, a particular type of delay differential equations that cannot be exponentially stable [1], [8].

This does not imply that repetitive control via delayed feedback is not achievable at all. Indeed, for the class of strictly positive real systems, a positive result was already presented by Ikeda and Takano [9]. By using the concept of hyperstability proposed by Popov, these authors proved that the tracking error of a control system of that class was bounded and that its square integral, computed on consecutive time intervals of length $T$ (the repetitive period), tends to zero. As is typical of the input-output approach, in their work there is no information on the asymptotic stability of the closed-loop full state. Another positive result can be found in [10], where, for positive real scalar systems, full state asymptotic stability is proven. In other words, it appears that, if one gives up with exponential stability, exact repetitive control with some kind of robustness (see [11, Th. 4.2, p. 26]) is achievable at least for some specific systems.

The investigation presented herein is a classical Lyapunov stability analysis based upon a novel invariance criteria of the type due to LaSalle and Barbashin-Krasovskii (see, e.g., [12, p. 108] or [6, p. $178]$ ). We first prove that any solution of the system under study is confined to a norm bounded set that in a reflexive Banach space is weakly compact [see, e.g., [13, pp. 338-339]], and next we show that the limit set in this bounded set coincides with the zero equilibrium point. We develop such an investigation in a Hilbert space equipped with the weak topology (see, e.g., [13, pp. 336-339]) by using the setting of dynamical systems defined on topological space (see, e.g., [14]). We initially prove weak convergence and then, on the basis of a wel- known property of compact operators, show that actually convergence is strong. In this way, overall closed-loop asymptotic stability in the Lyapunov sense is proved.

\section{REPETITIVE CONTROL}

Consider a finite-dimensional linear system

$$
\begin{array}{r}
\dot{x}(t)=A x(t)+B u(t), x(t) \in \Re^{n} \\
A \in \Re^{n \times n}, B \in \Re^{n \times m} \\
y(t)=C x(t), u(t), y(t) \in \Re^{m} \\
t \in[0, \infty), C \in \Re^{m \times n}
\end{array}
$$

where $x(t)$ is the plant state, $u(t)$ the control, and $y(t)$ the output, and the infinite-dimensional dynamic feedback

$$
u(t)=u(t-T)-\beta y(t), \beta \in(0, \infty), T \in \Re^{+}
$$

with initial conditions

$$
x(0)=x^{\circ}, u(s)=\varphi(s), s \in[-T, 0) .
$$

Let $L_{2}^{m}[0, T]$ denote the Hilbert space of vector valued square integrable functions on the interval $[0, T]$, equipped with the scalar product ${ }^{1}$

$$
\langle\varphi, \psi\rangle_{L_{2}^{m}}=\frac{1}{T} \int_{0}^{T} \varphi^{\prime}(s) \psi(s) d s, \varphi, \psi:[0, T] \rightarrow \Re^{m}
$$

${ }^{1}$ For the sake of clarity, when considering the scalar product and the norm on a given Hilbert space, we add its symbol as a subscript. 